\title{
ON THE STEINHAUS AND BERGMAN PROPERTIES FOR INFINITE PRODUCTS OF FINITE GROUPS
}

\author{
SIMON THOMAS \\ Mathematics Department, Rutgers University, \\ 110 Frelinghuysen Road, Piscataway, \\ NJ 08854-8019, USA \\ sthomas@math.rutgers.edu \\ JINDŘICH ZAPLETAL \\ Institute of Mathematics, Czech Academy of Sciences, \\ Prague, Czech Republic \\ and \\ Department of Mathematics, University of Florida, \\ Gainesville, FL 32611-8105, USA \\ zapletal@math.cas.cz \\ Received 15 February 2012 \\ Revised 22 July 2012 \\ Accepted 22 July 2012 \\ Published 27 September 2012
}

\begin{abstract}
We study the relationship between the existence of nonprincipal ultrafilters over $\omega$ and the failure of the automatic continuity, Steinhaus and Bergman properties for infinite products of finite groups.
\end{abstract}

Keywords: Ultrafilters; products of finite groups; automatic continuity.

AMS Subject Classification: 03E02, 03E25, 20A15

\section{Introduction}

In this paper, we will investigate the status of the Steinhaus and Bergman properties for infinite products of finite groups in various axiomatic frameworks. Our interest in these properties is partially motivated by the automatic continuity problem for Polish groups. More specifically, we will be interested in the question of which infinite products $G=\prod G_{n}$ of nontrivial finite groups have the automatic continuity property, i.e. have the property that every homomorphism $\varphi: G \rightarrow H$ from $G$ into a Polish group $H$ is necessarily continuous. In set theory with the Axiom of Choice, infinite products of finite groups typically fail to have this property; and, in fact, no examples of infinite products of finite groups with this property are 
currently known. The basic example of a discontinuous homomorphism involves a nonprincipal ultrafilter $\mathcal{U}$ over the set $\omega$ of natural numbers.

Example 1.1. Suppose that there exists a fixed nontrivial finite group $F$ such that $G_{n} \cong F$ for all $n \in \omega$. Then the corresponding ultraproduct $\prod_{\mathcal{U}} G_{n}$ is isomorphic to $F$ and the associated homomorphism $\varphi: \prod G_{n} \rightarrow F$ is not continuous, since $\operatorname{ker} \varphi$ is a dense subgroup and hence is not closed.

The automatic continuity property for some more interesting infinite products of finite groups can be shown to fail for more complicated reasons.

Example 1.2. Let $d \geq 2$ and suppose that $G_{n}=\operatorname{SL}\left(d, p_{n}\right)$, where $\left(p_{n} \mid n \in \omega\right)$ is an increasing sequence of primes. If $K=\prod_{\mathcal{U}} \mathbb{F}_{p_{n}}$ is the corresponding ultraproduct of the fields $\mathbb{F}_{p_{n}}$ of order $p_{n}$, then

$$
\prod_{\mathcal{U}} \mathrm{SL}\left(d, p_{n}\right) \cong \mathrm{SL}\left(d, \prod_{\mathcal{U}} \mathbb{F}_{p_{n}}\right)=\operatorname{SL}(d, K)
$$

and thus $\operatorname{SL}(d, K)$ is a homomorphic image of $\prod \operatorname{SL}\left(d, p_{n}\right)$. Since $K$ is a field of characteristic 0 and cardinality $2^{\aleph_{0}}$, it follows that $K$ embeds into $\mathbb{C}$ and hence $\mathrm{SL}(d, K)$ embeds into $\mathrm{SL}(d, \mathbb{C})$. (The fact that an ultraproduct of finite structures, if infinite, actually has cardinality $2^{\aleph_{0}}$ is due to Shelah [28].) Once again, it is clear that the associated homomorphism $\varphi: \prod \mathrm{SL}\left(d, p_{n}\right) \rightarrow \mathrm{SL}(d, \mathbb{C})$ is not continuous.

Remark 1.3. In Sec. 3, we will present a more sophisticated construction involving an embedding of $K$ into the field of Puiseux series over the field $\overline{\mathbb{Q}}$ of algebraic numbers, which yields a discontinuous homomorphism of $\prod \operatorname{SL}\left(d, p_{n}\right)$ into the infinite symmetric group $\operatorname{Sym}(\omega)$.

It is natural to ask whether the existence of a nonprincipal ultrafilter $\mathcal{U}$ over $\omega$ is either necessary or sufficient in the above constructions of discontinuous homomorphisms. (The existence of a nonprincipal ultrafilter $\mathcal{U}$ is clearly sufficient in Example 1.1. However, the construction in Example 1.2 also makes use of the existence of an embedding of the field $K=\prod_{\mathcal{U}} \mathbb{F}_{p_{n}}$ into $\mathbb{C}$ and the usual proofs of this result rely on the existence of transcendence bases for both $K$ and $\mathbb{C}$.) Of course, when considering this kind of question, we cannot work with the usual $Z F C$ axioms of set theory since these already imply the existence of nonprincipal ultrafilters over arbitrary infinite sets. Instead we will work with the axiom system $Z F+D C$, where $D C$ is the following weak form of the Axiom of Choice.

Axiom of Dependent Choice. (DC) Suppose that $X$ is a nonempty set and that $R$ is a binary relation on $X$ such that for all $x \in X$, there exists $y \in X$ with $x R y$. Then there exists a function $f: \omega \rightarrow X$ such that $f(n) R f(n+1)$ for all $n \in \omega$.

The axiom system $Z F+D C$ is sufficient to develop most of real analysis and descriptive set theory, but is insufficient to prove the existence of pathologies such 
as nonmeasurable sets. (For example, see Moschovakis [23].) In particular, since nonprincipal ultrafilters over $\omega$ are nonmeasurable when regarded as subsets of the Cantor space $2^{\mathbb{N}}$, it follows that $Z F+D C$ does not prove the existence of such ultrafilters.

We will provide a structured answer to the above question. Firstly, the following result is well known. (For example, see Rosendal [26, Sec. 2].)

Theorem 1.4. It is consistent with $Z F+D C$ that if $G, H$ are any Polish groups, then every homomorphism $\varphi: G \rightarrow H$ is continuous.

In fact, assuming the existence of suitable large cardinals, this is true in $L(\mathbb{R})$, the canonical minimal model of $Z F$ which contains all of the ordinals and all of the real numbers. Of course, this implies the well known result that $L(\mathbb{R})$ does not contain any nonprincipal ultrafilters over $\omega$. While it seems almost certain that the existence of a nonprincipal ultrafilter over $\omega$ is necessary to prove the failure of the automatic continuity property for suitably chosen infinite products $G=\prod G_{n}$ of finite groups, we have not completely settled this question. However, in Sec. 4, we will prove a number of partial results in this direction, including the following theorem.

Theorem 1.5. $(Z F+D C)$ Suppose that $d \geq 2$ and that $\left(p_{n} \mid n \in \omega\right)$ is an increasing sequence of primes. If there exists a discontinuous homomorphism $\varphi$ : $\prod \operatorname{SL}\left(d, p_{n}\right) \rightarrow \operatorname{Sym}(\omega)$, then there exists a nonprincipal ultrafilter over $\omega$.

On the other hand, we will show that the existence of a nonprincipal ultrafilter over $\omega$ is not sufficient to prove the failure of the automatic continuity property for suitably chosen infinite products of finite groups. In order to explain this result, it will be necessary in the remainder of this section to assume the existence of suitable large cardinals. We will not specify the precise large cardinal hypothesis that we need until it becomes necessary to do so in Sec. 7. (This paper has been written so that the first six sections can be read by mathematicians with no knowledge of advanced set theory, such as forcing, large cardinals, etc. It is only in the final section that some knowledge of advanced set theory is needed and this section can be omitted by mathematicians without the necessary background.) Following the usual convention [35], we will indicate the use of a large cardinal hypothesis by writing $(L C)$ before the statement of the relevant theorem. The following result is a special case of a more general result that we will present in Sec. 5 .

Theorem 1.6. (LC) It is consistent with $Z F+D C$ that

(i) there exists a nonprincipal ultrafilter $\mathcal{U}$ over $\omega$; and

(ii) for each $d \geq 2$, if $\left(p_{n} \mid n \in \omega\right)$ is a sufficiently fast growing sequence of primes, then $\prod \mathrm{SL}\left(d, p_{n}\right)$ has the automatic continuity property.

In fact, assuming the existence of suitable large cardinals, this is true in $L(\mathbb{R})[\mathcal{U}]$, the minimal model of $Z F$ containing all of the ordinals and real numbers, together 
with a Ramsey ultrafilter $\mathcal{U}$ over $\omega$. Under a suitable large cardinal hypothesis, $L(\mathbb{R})[\mathcal{U}]$ has canonicity features parallel to those of $L(\mathbb{R})$; and, in particular, its theory does not depend on the choice of the Ramsey ultrafilter $\mathcal{U}$. Di PriscoTodorcevic [7] have shown that many of the regularity properties of $L(\mathbb{R})$ continue to hold in $L(\mathbb{R})[\mathcal{U}]$. For example, in $L(\mathbb{R})[\mathcal{U}]$, every uncountable set of reals has a perfect subset. Thus it seems natural to regard $L(\mathbb{R})[\mathcal{U}]$ as a canonical model of $Z F+D C$ in which a minimal number of the pathological consequences of the Axiom of Choice holds, modulo the existence of a nonprincipal ultrafilter $\mathcal{U}$ over $\omega$. The results of this paper provide yet more evidence for this point of view.

Up until this point, we have considered two examples of infinite products of finite groups; namely, infinite products of a fixed finite group $F$ and infinite products of the form $\prod \mathrm{SL}\left(d, p_{n}\right)$ for various increasing sequences $\left(p_{n} \mid n \in \omega\right)$ of primes. In the first example, we have seen that the existence of a nonprincipal ultrafilter $\mathcal{U}$ over $\omega$ is sufficient to prove the failure of the automatic continuity property; while in the second example, this is not sufficient. Now we should also consider a third example; namely, the infinite product $\prod \operatorname{Alt}(n)$ of the finite alternating groups. In this case, as we will explain in Sec. 6, it is natural to conjecture that the automatic continuity property does hold. So what is the essential difference between these three examples? Perhaps surprisingly, the key to our analysis of the infinite product $\prod G_{n}$ of finite groups turns out to be the "asymptotic representation theory" of the sequence $\left(G_{n} \mid n \in \omega\right)$. In order to state this more precisely, it is necessary to introduce the following definitions.

Definition 1.7. Let $H$ be a nontrivial finite group.

(i) If $K$ is a field, then $d_{K}(H)$ denotes the minimal dimension of a nontrivial $K$-representation of $H$; i.e. the least $d$ such that there exists a nontrivial homomorphism $\theta: H \rightarrow \mathrm{GL}(d, K)$.

(ii) $d(H)=\min \left\{d_{K}(H) \mid K\right.$ is a field $\}$.

Example 1.8. Suppose that $p \geq 5$ is a prime and that $H=\mathrm{SL}(d, p)$.

(i) If $d=2$, then $d_{\mathbb{C}}(H)=(p-1) / 2$ and $d(H)=2$.

(ii) If $d>2$, then $d_{\mathbb{C}}(H)=\left(p^{d}-p\right) /(p-1)$ and $d(H)=d$.

(For example, see Humphreys [12] and Tiep-Zalesskii [32].)

Example 1.9. If $n>8$ and $H=\operatorname{Alt}(n)$, then $d_{\mathbb{C}}(H)=n-1$ and $d(H)=n-2$. (For example, see James [14] and Wagner [33, 34].)

Suppose that $\left(G_{n} \mid n \in \omega\right)$ is an arbitary sequence of nontrivial finite groups. Then we will prove that:

(a) if $\lim \inf d\left(G_{n}\right)<\infty$, then $\prod G_{n}$ does not have the automatic continuity property in the actual set-theoretic universe $V$. 
It seems reasonable to conjecture that the converse of (a) also holds, perhaps after imposing some suitable algebraic conditions (such as simplicity) on the groups $G_{n}$. In particular, it seems likely that $\prod \operatorname{Alt}(n)$ has the automatic continuity property in $V$. Now suppose that $\lim \inf d\left(G_{n}\right)<\infty$. Then we will prove that:

(b) if $\liminf d_{\mathbb{C}}\left(G_{n}\right)<\infty$, then the existence of a nonprincipal ultrafilter $\mathcal{U}$ over $\omega$ is enough to prove that $\prod G_{n}$ does not have the automatic continuity property.

On the other hand, if the groups $G_{n}$ are members of a suitably chosen class $\mathcal{C}$, such as the class of groups of the form $\operatorname{SL}(d, p)$ for some fixed $d \geq 2$, then we will prove that:

(c) assuming $(L C)$, if $\left(d_{\mathbb{C}}\left(G_{n}\right) \mid n \in \omega\right)$ grows sufficiently fast, then $\prod G_{n}$ has the automatic continuity property in $L(\mathbb{R})[\mathcal{U}]$.

This paper is organized as follows. In Sec. 2, we will discuss the Steinhaus and Bergman properties for infinite products of finite groups. In Sec. 3, working with the usual $Z F C$ axioms of set theory, we will prove that the Steinhaus and Bergman properties fail for various infinite products of finite groups. In Sec. 4, working with the axiom system $Z F+D C$, we will prove that the failure of the Bergman property for suitably chosen infinite products of finite groups implies the existence of a nonprincipal ultrafilter over $\omega$; and we will show that the failure of a weak form of the Steinhaus property also implies the existence of such an ultrafilter. In Sec. 5, we will present a partition property $P P$ for products of finite sets with measures; and we will show that $Z F+D C+P P$ implies that various infinite products of finite groups have both the Bergman property and the Steinhaus property. In Sec. 6, we will briefly discuss the questions of which infinite products of nonabelian finite simple groups have either the Bergman property or the Steinhaus property in the actual set-theoretic universe $V$. Finally, in Sec. 7, assuming the existence of suitable large cardinals, we will prove that $L(\mathbb{R})[\mathcal{U}]$ satisfies $P P$.

Notation 1.10. Let $\left(H_{n} \mid n \in \omega\right)$ be a sequence of finite groups and let $H=\prod H_{n}$. Suppose that $A \subseteq \omega$.

(i) $\prod_{n \in A} H_{n}$ denotes the subgroup of $H$ consisting of those elements $\left(h_{n}\right) \in H$ such that $h_{n}=1$ for all $n \in \omega \backslash A$.

(ii) If $h=\left(h_{n}\right) \in H$, then $h \uparrow A$ denotes the element $\left(g_{n}\right) \in \prod_{n \in A} H_{n}$ such that $g_{n}=h_{n}$ for all $n \in A$.

Recall that $H=\prod H_{n}$ is a Polish topological group with neighborhood basis of the identity given by $\left\{\prod_{n \in A} H_{n} \mid A\right.$ is a cofinal subset of $\left.\omega\right\}$.

Suppose that $U$ is a subset of the group $G$. Then for each $t \geq 1, U^{t}$ denotes the set of elements $g \in G$ which can be expressed as a product $g=u_{1} \cdots u_{t}$, where each $u_{i} \in U$. The subset $U$ is said to be symmetric if $U=U^{-1}$ is closed under taking inverses. 


\section{The Steinhaus and Bergman Properties}

In this section, we will discuss the Steinhaus and Bergman properties for infinite products of finite groups. The Steinhaus property was introduced by RosendalSolecki [25] in the context of the automatic continuity problem for homomorphisms between topological groups. In the following definition, a subset $W$ of a group $G$ is said to be countably syndetic if there exist elements $g_{n} \in G$ for $n \in \omega$ such that $G=\bigcup_{n \in \omega} g_{n} W$.

Definition 2.1. Let $G$ be a topological group. Then $G$ has the Steinhaus property if there exists a fixed integer $k \geq 1$ such that for every symmetric countably syndetic subset $W \subseteq G$, the $k$-fold product $W^{k}$ contains an open neighborhood of the identity element $1_{G}$.

Proposition 2.2. (Rosendal-Solecki [25]) If $G$ is a topological group with the Steinhaus property and $\varphi: G \rightarrow H$ is a homomorphism into a separable group $H$, then $\varphi$ is necessarily continuous.

The class of groups with the Steinhaus property includes Polish groups with ample generics $[16], \operatorname{Aut}(\mathbb{Q},<), \operatorname{Homeo}(\mathbb{R})[25]$ and full groups of ergodic countable Borel equivalence relations [17]. However, no infinite product of finite groups is currently known to have the Steinhaus property. Of course, by Example 1.2 and Proposition 2.2, it follows that if $\left(p_{n} \mid n \in \omega\right)$ is an increasing sequence of primes and $d \geq 2$, then $\prod \mathrm{SL}\left(d, p_{n}\right)$ does not have the Steinhaus property. We will prove the following more general result in Sec. 3 .

Theorem 2.3. Suppose that $\left(G_{n} \mid n \in \omega\right)$ is a sequence of nontrivial finite groups. If $\lim \inf d\left(G_{n}\right)<\infty$, then $\prod G_{n}$ does not have the automatic continuity property and hence does not have the Steinhaus property.

As the reader has probably guessed, the proof of Theorem 2.3 involves the use of a suitable ultraproduct $\prod_{\mathcal{U}} G_{n}$. However, the following strengthening of Theorem 1.6, which we will prove in Sec. 5, shows that the existence of a nonprincipal ultrafilter over $\omega$ is not always enough to prove that such a product $\prod G_{n}$ does not have the Steinhaus property.

Theorem 2.4. (LC) It is consistent with $Z F+D C$ that

(i) there exists a nonprincipal ultrafilter $\mathcal{U}$ over $\omega$; and

(ii) for each $d \geq 2$, if $\left(p_{n} \mid n \in \omega\right)$ is a sufficiently fast growing sequence of primes, then $\prod \mathrm{SL}\left(d, p_{n}\right)$ has the Steinhaus property.

Once again, assuming the existence of suitable large cardinals, this is true in $L(\mathbb{R})[\mathcal{U}]$, the minimal model of $Z F$ containing all of the ordinals and real numbers, together with a Ramsey ultrafilter $\mathcal{U}$ over $\omega$. 
The Bergman property was introduced by Bergman [2] as a strengthening of the notion of uncountable cofinality which was introduced earlier by MacphersonNeumann [21].

Definition 2.5. Suppose that $G$ is a non-finitely generated group.

(a) $G$ has countable cofinality if $G=\bigcup_{n \in \omega} G_{n}$ can be expressed as the union of a countable increasing chain of proper subgroups. Otherwise, $G$ has uncountable cofinality.

(b) $G$ is Cayley bounded if for every symmetric generating set $S$, there exists an integer $n \geq 1$ such that every element $g \in G$ can be expressed as a product $g=s_{1} \cdots s_{n}$, where each $s_{i} \in S \cup\{1\}$.

(c) $G$ has the Bergman property if $G$ has uncountable cofinality and is Cayley bounded.

By de Cornulier [6], a group $G$ has the Bergman property if and only if whenever $G$ acts isometrically on a metric space, every $G$-orbit has a finite diameter. For this reason, groups with the Bergman property are often said to be "strongly bounded". The class of groups with the Bergman property includes the symmetric groups over infinite sets [2], automorphism groups of various infinite structures [9, 13] and oligomorphic groups with ample generics [16]. The following easy observation is essentially contained in Bergman [2, Lemma 10].

Lemma 2.6. If $G$ is a non-finitely generated group, then the following conditions are equivalent.

(a) G has the Bergman property.

(b) If $G=\bigcup_{n \in \omega} U_{n}$ is the union of an increasing chain of symmetric subsets such that $U_{n} U_{n} \subseteq U_{n+1}$ for all $n \in \omega$, then there exists an $n \in \omega$ such that $U_{n}=G$.

In [6], improving an earlier result of Koppelberg-Tits [18], de Cornulier proved that if $G$ is a product of infinitely many copies of a fixed finite perfect group, then $G$ has the Bergman property; and Zalan Gyenis has recently checked that the arguments of Saxl-Shelah-Thomas [27] can be modified to prove that an infinite product $\prod H_{n}$ of finite simple groups has the Bergman property if and only if $\prod H_{n}$ has uncountable cofinality. This yields an explicit classification of the infinite products $\prod H_{n}$ of finite simple groups satisfying the Bergman property, which we will discuss in Sec. 6. On the other hand, there are many infinite products of finite groups which are known not to have the Bergman property. In particular, the following result holds.

Theorem 2.7. If $d \geq 2$ and $\left(p_{n} \mid n \in \omega\right)$ is an increasing sequence of primes, then:

(a) $\prod \mathrm{SL}\left(d, p_{n}\right)$ has countable cofinality; and

(b) $\prod \mathrm{SL}\left(d, p_{n}\right)$ is not Cayley bounded. 
Theorem 2.7(a) is essentially contained in Saxl-Shelah-Thomas [27]. However, for the sake of completeness, we will quickly sketch the very easy proof. (We will present the proof of Theorem 2.7(b) in Sec. 3.) Let $\mathcal{U}$ be a nonprincipal ultrafilter over $\omega$ and let $K=\prod_{\mathcal{U}} \mathbb{F}_{p_{n}}$ be the corresponding ultraproduct of the fields $\mathbb{F}_{p_{n}}$ of order $p_{n}$. Then $K$ is an uncountable field and

$$
\prod_{\mathcal{U}} \mathrm{SL}\left(d, p_{n}\right) \cong \mathrm{SL}\left(d, \prod_{\mathcal{U}} \mathbb{F}_{p_{n}}\right)=\operatorname{SL}(d, K) .
$$

It follows that $\mathrm{SL}(d, K)$ is a homomorphic image of $\prod \mathrm{SL}\left(d, p_{n}\right)$ and hence Theorem 2.7(a) is an immediate consequence of the following observation.

Proposition 2.8. If $F$ is an uncountable field, then $\operatorname{SL}(d, F)$ has countable cofinality.

Proof. Let $B$ be a transcendence basis of $F$ over its prime subfield. Then $B$ is uncountable and hence we can express $B=\bigcup_{n \in \omega} B_{n}$ as the union of a countable strictly increasing chain of proper subsets. For each $n \in \omega$, let $F_{n}$ be the algebraic closure of $B_{n}$ in $F$. Then the strictly increasing chain of proper subgroups

$$
\mathrm{SL}(d, F)=\bigcup_{n \in \omega} \mathrm{SL}\left(d, F_{n}\right)
$$

witnesses that $\mathrm{SL}(d, F)$ has countable cofinality.

The following result, which will be proved in Sec. 4, shows that the existence of a nonprincipal ultrafilter over $\omega$ is necessary in order to prove either Theorem 2.7(a) or Theorem 2.7(b).

Theorem 2.9. $(Z F+D C)$ Let $d \geq 2$ and let $\left(p_{n} \mid n \in \omega\right)$ be an increasing sequence of primes. If $\prod \mathrm{SL}\left(d, p_{n}\right)$ does not have the Bergman property, then there exists a nonprincipal ultrafilter over $\omega$.

On the other hand, we will also show that the existence of a nonprincipal ultrafilter over $\omega$ is not sufficient to prove either of the parts of Theorem 2.7.

Theorem 2.10. (LC) If $d \geq 2$ and $\left(p_{n} \mid n \in \omega\right)$ is a sufficiently fast growing sequence of primes, then $\prod \mathrm{SL}\left(d, p_{n}\right)$ has the Bergman property in $L(\mathbb{R})[\mathcal{U}]$.

Examining the above proof of Theorem 2.7(a), we see that it relies upon the following three consequences of the Axiom of Choice:

(i) the existence of a nonprincipal ultrafilter $\mathcal{U}$ over $\omega$;

(ii) the existence of a transcendence basis $B$ of the field $\prod_{\mathcal{U}} \mathbb{F}_{p_{n}}$; and

(iii) the existence of an expression of $B$ as the union of a countable strictly increasing chain of proper subsets. 
Clearly $L(\mathbb{R})[\mathcal{U}]$ satisfies (i); and since $D C$ implies that every infinite set has a denumerably infinite subset, it follows easily that every infinite set can be expressed as the union of a countable strictly increasing chain of proper subsets in $L(\mathbb{R})[\mathcal{U}$. Consequently, assuming $L C$, if $\left(p_{n} \mid n \in \omega\right)$ is a sufficiently fast growing sequence of primes, then (ii) must fail in $L(\mathbb{R})[\mathcal{U}]$.

Corollary 2.11. (LC) If $\left(p_{n} \mid n \in \omega\right)$ is a sufficiently fast growing sequence of primes, then the field $\prod_{\mathcal{U}} \mathbb{F}_{p_{n}}$ does not have a transcendence basis in $L(\mathbb{R})[\mathcal{U}]$.

\section{On the Failure of the Bergman and Steinhaus Properties}

In this section, we will first show that if $\left(p_{n} \mid n \in \omega\right)$ is an increasing sequence of primes and $d \geq 2$, then:

- There exists a discontinuous homomorphism of $\prod \operatorname{SL}\left(d, p_{n}\right)$ into $\operatorname{Sym}(\omega)$.

- $\prod \mathrm{SL}\left(d, p_{n}\right)$ is not Cayley bounded.

Then we will prove that if $\left(G_{n} \mid n \in \omega\right)$ is a sequence of nontrivial finite groups such that $\lim \inf d\left(G_{n}\right)<\infty$, then $\prod G_{n}$ does not have the automatic continuity property and hence does not have the Steinhaus property.

Once again, let $\mathcal{U}$ be a nonprincipal ultrafilter over $\omega$ and let $K=\prod_{\mathcal{U}} \mathbb{F}_{p_{n}}$ be the corresponding ultraproduct of the fields $\mathbb{F}_{p_{n}}$ of order $p_{n}$. Our arguments depend upon the existence of a suitable valuation $v: K \rightarrow \mathbb{Q} \cup\{\infty\}$.

Definition 3.1. Let $F$ be a field and let $t$ be an indeterminate over $F$. Then $F((t))$ denotes the corresponding field of formal power series; and

$$
\mathbf{P}(F)=\bigcup_{n \geq 1} F\left(\left(t^{1 / n}\right)\right)
$$

denotes the corresponding field of Puiseux series. Let $v_{F}: \mathbf{P}(F) \rightarrow \mathbb{Q} \cup\{\infty\}$ be the valuation such that if

$$
0 \neq a=\sum_{k \geq M}^{\infty} a_{k} t^{k / n} \in \mathbf{P}(F),
$$

where $a_{k} \in F, a_{M} \neq 0, k, M \in \mathbb{Z}$ and $n \geq 1$, then $v_{F}(a)=M / n$. (As usual, we set $v_{F}(0)=\infty$.)

It is well known that if $F$ is an algebraically closed field of characteristic 0 , then $\mathbf{P}(F)$ is algebraically closed. (For example, see Chevalley [4].) In particular, if $\overline{\mathbb{Q}}$ is the field of algebraic numbers, then $\mathbf{P}(\overline{\mathbb{Q}})$ is an algebraically closed field of cardinality $2^{\aleph_{0}}$. Hence, since $K=\prod_{\mathcal{U}} \mathbb{F}_{p_{n}}$ is a field of characteristic 0 and cardinality $2^{\aleph_{0}}$, we can assume that $K$ is a subfield of $\mathbf{P}(\overline{\mathbb{Q}})$. Furthermore, since $K$ is uncountable and the automorphism group of $\mathbf{P}(\overline{\mathbb{Q}})$ acts transitively on nonalgebraic elements, we can assume that $t \in K$. From now on, we let $v=v_{\overline{\mathbb{Q}}} \uparrow K$ denote the corresponding valuation of $K$ and let $R=\{a \in K \mid v(a) \geq 0\}$ be the 
corresponding valuation ring. We will make use of the following result, which was proved in Thomas [31, Sec. 2].

Theorem 3.2. $[\mathrm{SL}(d, K): \mathrm{SL}(d, R)]=\omega$.

Corollary 3.3. There exists a discontinuous homomorphism of $\prod \mathrm{SL}\left(d, p_{n}\right)$ into $\operatorname{Sym}(\omega)$.

Proof. Let $\pi: \prod \mathrm{SL}\left(d, p_{n}\right) \rightarrow \mathrm{SL}(d, K)$ be the canonical surjective homomorphism and let $H=\pi^{-1}(\operatorname{SL}(d, R))$. Then $\left[\prod \operatorname{SL}\left(d, p_{n}\right): H\right]=\omega$ and the action of $\prod \mathrm{SL}\left(d, p_{n}\right)$ on the cosets of $H$ induces a homomorphism

$$
\varphi: \prod \mathrm{SL}\left(d, p_{n}\right) \rightarrow \operatorname{Sym}(\omega)
$$

such that $\varphi(H)$ is the stabilizer of 0 in $\varphi\left(\prod \operatorname{SL}\left(d, p_{n}\right)\right)$. If $S$ is the stabilizer of 0 in $\operatorname{Sym}(\omega)$, then $S$ is an open subgroup of $\operatorname{Sym}(\omega)$ and $\varphi^{-1}(S)=H$. Since $H$ is clearly not an open subgroup of $\prod \operatorname{SL}\left(d, p_{n}\right)$, it follows that $\varphi$ is not continuous.

Next we will prove that $\prod \mathrm{SL}\left(d, p_{n}\right)$ is not Cayley bounded. By the following easy observation, it is enough to show that $\mathrm{SL}(d, K)$ is not Cayley bounded.

Lemma 3.4. Suppose that $G$ is a group and that $N \unlhd G$ is a normal subgroup. If $G$ is Cayley bounded, then $H=G / N$ is also Cayley bounded.

Proof. Suppose that the symmetric generating set $S \subseteq H$ witnesses that $H$ is not Cayley bounded. Let $\pi: G \rightarrow H$ be the canonical surjective homomorphism and let $T=\pi^{-1}(S)$. Then $T$ witnesses that $G$ is not Cayley bounded.

From now on, in order to simplify notation, we will assume that $d=2$. Recall that after identifying $K$ with its image under a suitable embedding into the field $\mathbf{P}(\overline{\mathbb{Q}})$ of Puiseux series in the indeterminate $t$, we have that $t \in K$. Also note that $v(t)=1$ and that $v\left(t^{-1}\right)=-1$. For each $k \in K^{*}=K \backslash\{0\}$, let

$$
x(k)=\left(\begin{array}{cc}
1 & k \\
0 & 1
\end{array}\right) \quad y(k)=\left(\begin{array}{cc}
1 & 0 \\
k & 1
\end{array}\right) \quad d(k)=\left(\begin{array}{cc}
k & 0 \\
0 & k^{-1}
\end{array}\right) .
$$

Then it is well known that $T=\left\{x(k) \mid k \in K^{*}\right\} \cup\left\{y(k) \mid k \in K^{*}\right\}$ generates $\mathrm{SL}(2, K)$. (For example, see Lang [19, Lemma XIII.8.1].) Let

$$
U=\left\{d(t), d\left(t^{-1}\right)\right\} \cup\{x(k) \mid 0 \leq v(k) \leq 2\} \cup\{y(k) \mid 0 \leq v(k) \leq 2\} .
$$

Since $v(-k)=v(k)$ for all $k \in K$, it follows that $U$ is a symmetric subset of $\mathrm{SL}(2, K)$. We claim that $U$ generates $\mathrm{SL}(2, K)$. To see this, note that

$$
d(t) x(k) d(t)^{-1}=x\left(t^{2} k\right), \quad d(t)^{-1} x(k) d(t)=x\left(t^{-2} k\right)
$$

and that

$$
v\left(t^{2} k\right)=v\left(t^{2}\right)+v(k)=v(k)+2, \quad v\left(t^{-2} k\right)=v\left(t^{-2}\right)+v(k)=v(k)-2 .
$$


Hence if $k \in K^{*}$, then there exists $m \in \mathbb{Z}$ such that $d(t)^{m} x(k) d(t)^{-m} \in U$; and similarly, there exists $m \in \mathbb{Z}$ such that $d(t)^{m} y(k) d(t)^{-m} \in U$. It follows that $T \subseteq$ $\langle U\rangle$ and hence $\langle U\rangle=\mathrm{SL}(2, K)$. Next for each matrix

$$
A=\left(\begin{array}{ll}
a_{1} & a_{2} \\
a_{3} & a_{4}
\end{array}\right) \in \mathrm{SL}(2, K)
$$

we define

$$
\tau(A)=\min \left\{v\left(a_{i}\right) \mid 1 \leq i \leq 4\right\}
$$

Notice that since

$$
\left(\begin{array}{ll}
a_{1} & a_{2} \\
a_{3} & a_{4}
\end{array}\right)\left(\begin{array}{ll}
b_{1} & b_{2} \\
b_{3} & b_{4}
\end{array}\right)=\left(\begin{array}{ll}
a_{1} b_{1}+a_{2} b_{3} & a_{1} b_{2}+a_{2} b_{4} \\
a_{3} b_{1}+a_{4} b_{3} & a_{3} b_{2}+a_{4} b_{4}
\end{array}\right)
$$

and since, for example,

$$
\begin{aligned}
v\left(a_{1} b_{1}+a_{2} b_{3}\right) & \geq \min \left\{v\left(a_{1} b_{1}\right), v\left(a_{2} b_{3}\right)\right\} \\
& =\min \left\{v\left(a_{1}\right)+v\left(b_{1}\right), v\left(a_{2}\right)+v\left(b_{3}\right)\right\},
\end{aligned}
$$

it follows that $\tau(A B) \geq \tau(A)+\tau(B)$ for all $A, B \in \mathrm{SL}(2, K)$. Finally recall that for each $m \in \mathbb{N}$, we have that $v\left(t^{-m}\right)=-m$ and so $\tau\left(d\left(t^{m}\right)\right)=-m$. It now follows easily that for each $n \in \mathbb{N}$, there exists $m \in \mathbb{N}$ such that $d\left(t^{m}\right)$ is not a product of $n$ elements of $U \cup\{1\}$. Thus $\operatorname{SL}(2, K)$ is not Cayley bounded and it follows that $\prod \mathrm{SL}\left(2, p_{n}\right)$ is also not Cayley bounded.

The remainder of this section is devoted to the proof of Theorem 2.3. Suppose that $\left(G_{n} \mid n \in \omega\right)$ is a sequence of nontrivial finite groups with $\lim \inf d\left(G_{n}\right)<\infty$. Then there exists an infinite subset $I \subseteq \omega$ and a fixed $d \geq 1$ such that for each $n \in I$ there exists a nontrivial homomorphism

$$
\varphi_{n}: G_{n} \rightarrow \mathrm{GL}\left(d, F_{n}\right)
$$

for some field $F_{n}$. In order to simplify notation, we will assume that $I=\omega$. Let $\mathcal{U}$ be a nonprincipal ultrafilter over $\omega$ and let

$$
\varphi: \prod_{\mathcal{U}} G_{n} \rightarrow \prod_{\mathcal{U}} \mathrm{GL}\left(d, F_{n}\right)
$$

be the homomorphism defined by $\left(g_{n}\right) \mathcal{U} \mapsto\left(\varphi_{n}\left(g_{n}\right)\right)_{\mathcal{U}}$. Let $F=\prod_{\mathcal{U}} F_{n}$ and $H=$ $\varphi\left(\prod_{\mathcal{U}} G_{n}\right)$. By Thomas [31, Theorem 2.1], since $F$ is a field of cardinality at most $2^{\aleph_{0}}$ and

$$
1 \neq H \leq \prod_{\mathcal{U}} \mathrm{GL}\left(d, F_{n}\right) \cong \mathrm{GL}(d, F)
$$

it follows that there exists a proper subgroup $H_{0}<H$ such that $1<\left[H: H_{0}\right] \leq \omega$. (As with our earlier arguments, the proof of Thomas [31, Theorem 2.1] involves defining a suitable valuation on $F$.) Let $L=\varphi^{-1}\left(H_{0}\right)$. Then $L$ is a proper subgroup of $\prod_{\mathcal{U}} G_{n}$ of countable (possibly finite) index. Let $\pi: \prod G_{n} \rightarrow \prod_{\mathcal{U}} G_{n}$ be the 
canonical surjective homomorphism and let $M=\pi^{-1}(L)$. Then $M$ is a proper subgroup of $\prod_{\mathcal{U}} G_{n}$ of countable (possibly finite) index. If $M$ has infinite index, then arguing as in the proof of Corollary 3.3, it follows that there exists a discontinuous homomorphism of $\prod G_{n}$ into $\operatorname{Sym}(\omega)$. Hence we can assume that $\left[\prod G_{n}: M\right]=$ $\ell>1$ is finite. Since $\mathcal{U}$ is nonprincipal, it follows that if $P$ is any open subgroup of $\prod G_{n}$, then $\pi(P)=\prod_{\mathcal{U}} G_{n}$. In particular, $M$ is not an open subgroup and hence there exists a discontinuous homomorphism from $\prod G_{n}$ into the finite group $\operatorname{Sym}(\ell)$. This completes the proof of Theorem 2.3.

\section{On the Existence of Nonprincipal Ultrafilters}

In this section, working with the axiom system $Z F+D C$, we will prove that the failure of the Bergman property for suitably chosen infinite products $\prod H_{n}$ of finite groups implies the existence of a nonprincipal ultrafilter over $\omega$. It is currently not known whether failures of the Steinhaus property also imply the existence of a nonprincipal ultrafilter over $\omega$. However, we will show that failures of a weak form of the Steinhaus property do indeed imply the existence of such an ultrafilter.

Theorem 4.1. $(Z F+D C)$ Let $\left(H_{n} \mid n \in \omega\right)$ be a sequence of nontrivial finite groups which satisfies the following condition:

(†) There is a fixed integer $t \geq 1$ such that for all $n \in \omega$, there is a conjugacy class $C_{n} \subseteq H_{n}$ such that $C_{n}^{t}=H_{n}$.

If $\prod H_{n}$ does not have the Bergman property, then there exists a nonprincipal ultrafilter over $\omega$.

Proof. Suppose that $G=\prod H_{n}$ does not have the Bergman property. Then we can express $G=\bigcup_{k \in \omega} U_{k}$ as the union of a strictly increasing chain of symmetric proper subsets such that $U_{k} U_{k} \subseteq U_{k+1}$ for all $k \in \omega$. Consider

$$
\mathcal{I}=\left\{A \subseteq \omega \mid \prod_{n \in A} H_{n} \subseteq U_{k} \text { for some } k \in \omega\right\} .
$$

Then clearly $\mathcal{I}$ is an ideal which contains all the finite subsets of $\omega$. Hence it is enough to prove that there exists a set $B \notin \mathcal{I}$ such that $\mathcal{I} \cap \mathcal{P}(B)$ is a prime ideal over $B$.

Suppose that no such set $B$ exists. Then for each $A \notin \mathcal{I}$, there exists $A^{\prime} \subseteq A$ such that $A^{\prime} \notin \mathcal{I}$ and $A \backslash A^{\prime} \notin \mathcal{I}$; and hence we can inductively find pairwise disjoint subsets $\left\{A_{k} \mid k \in \omega\right\}$ of $\omega$ such that $A_{k} \notin \mathcal{I}$ and $\omega \backslash \bigcup_{\ell \leq k} A_{\ell} \notin \mathcal{I}$ for all $k \in \omega$.

Claim 4.2. There exists $k \in \omega$ such that for every $h \in \prod_{n \in A_{k}} H_{n}$, there exists $g \in U_{k}$ such that $g\left\lceil A_{k}=h\right.$.

Proof of Claim 4.2. If not, then there exists $h \in G$ such that for all $k \in \omega$ and $g \in U_{k}$, we have that $g \uparrow A_{k} \neq h \uparrow A_{k}$. But this means that $h \notin \bigcup_{k \in \omega} U_{k}$, which is a contradiction. 
Fix some such $k \in \omega$. For each $n \in A_{k}$, let $C_{n}$ be the conjugacy class of $H_{n}$ given by condition $(\dagger)$ and let $h=\left(h_{n}\right) \in \prod_{n \in A_{k}} H_{n}$ be such that $h_{n} \in C_{n}$ for all $n \in A_{k}$. Let $h \in U_{\ell}$ and let $m=\max \{k, \ell\}$. Then it follows that the conjugacy class $C$ of $h$ in $\prod_{n \in A_{k}} H_{n}$ is contained in $U_{m}^{3}$; and hence $\prod_{n \in A_{k}} H_{n}$ is contained in $U_{m}^{3 t}$. But this means that $\prod_{n \in A_{k}} H_{n} \subseteq U_{s}$ for some $s \geq m$, which contradicts the fact that $A_{k} \notin \mathcal{I}$. This completes the proof of Theorem 4.1 .

Clearly Theorem 2.9 is an immediate consequence of Theorem 4.1, together with the following result.

Proposition 4.3. (Ellers-Gordeev-Herzog [10]) Suppose that $K$ is any field such that $|K|>5$ and that $C$ is any noncentral conjugacy class of $\mathrm{SL}(d, K)$.

(i) If $d=2$, then $C^{8}=\mathrm{SL}(2, K)$.

(ii) If $d>2$, then $C^{2 d}=\mathrm{SL}(d, K)$.

In the remainder of this section, we will consider the following weak form of the Steinhaus property.

Definition 4.4. The Polish group $G$ is said to have the weak Steinhaus property if for every symmetric countably syndetic subset $W \subseteq G$, there exists an integer $k \geq 1$ such that $W^{k}$ contains an open neighborhood of the identity element $1_{G}$.

For example, if the Polish group $G$ has a non-open subgroup of countable index, then clearly $G$ does not have the weak Steinhaus property. In particular, if we work with $Z F C$, then the results of Sec. 3 show that $\prod \operatorname{SL}\left(d, p_{n}\right)$ does not have the weak Steinhaus property. The rest of this section is devoted to the proof of the following result.

Theorem 4.5. $(Z F+D C)$ Suppose that $d \geq 2$ and that $\left(p_{n} \mid n \in \omega\right)$ is an increasing sequence of primes. If $\prod \mathrm{SL}\left(d, p_{n}\right)$ does not have the weak Steinhaus property, then there exists a nonprincipal ultrafilter over $\omega$.

Notice that Theorem 1.5 is an easy consequence of Theorem 4.5. For assume that $\varphi: \prod \operatorname{SL}\left(d, p_{n}\right) \rightarrow \operatorname{Sym}(\omega)$ is a discontinuous homomorphism. Then there exists an open subgroup $U \leq \operatorname{Sym}(\omega)$ such that $\varphi^{-1}(U)$ is not open in $\prod \operatorname{SL}\left(d, p_{n}\right)$. Since $U$ has countable index in $\operatorname{Sym}(\omega)$, it follows that $\varphi^{-1}(U)$ is a non-open subgroup of countable index in $\prod \mathrm{SL}\left(d, p_{n}\right)$ and hence $\prod \mathrm{SL}\left(d, p_{n}\right)$ does not have the weak Steinhaus property.

Most of our effort will go into proving the following special case of Theorem 4.5.

Theorem 4.6. $(Z F+D C)$ Suppose that $d \geq 2$ and that $\left(p_{n} \mid n \in \omega\right)$ is an increasing sequence of primes. If there exists a subgroup $H<\prod \operatorname{SL}\left(d, p_{n}\right)$ such that $\left[\Pi \operatorname{SL}\left(d, p_{n}\right): H\right]=\omega$, then there exists a nonprincipal ultrafilter over $\omega$.

The proof of Theorem 4.6 makes use of some of the basic properties of primitive permutation groups. Recall that if $\Omega$ is any nonempty set and $G \leq \operatorname{Sym}(\Omega)$, then 
$G$ is said to act primitively on $\Omega$ if:

(i) $G$ acts transitively on $\Omega$; and

(ii) there does not exist a nontrivial $G$-invariant equivalence relation on $\Omega$.

It is well known that if $G \leq \operatorname{Sym}(\Omega)$ is a transitive subgroup, then $G$ acts primitively on $\Omega$ if and only if the stabilizer $G_{\alpha}=\{g \in G \mid g(\alpha)=\alpha\}$ is a maximal subgroup of $G$ for some (equivalently every) $\alpha \in \Omega$. Also if $G$ acts primitively on $\Omega$ and $1 \neq N \unlhd G$ is a nontrivial normal subgroup, then it follows that $N$ must act transitively on $\Omega$. (For example, see Cameron [3, Theorem 1.7].)

The proof of Theorem 4.6 also makes use of the following easy consequence of Proposition 4.3.

Lemma 4.7. $(Z F+D C)$ Suppose that $d \geq 2$ and that $\left(p_{n} \mid n \in \omega\right)$ is an increasing sequence of primes. Then every normal subgroup $N$ of countable index in $\prod \operatorname{SL}\left(d, p_{n}\right)$ is open.

Proof. Let $G=\prod \mathrm{SL}\left(d, p_{n}\right)$ and let $\mathcal{F}=\left\{g_{\tau}=\left(g_{\tau}(n)\right) \mid \tau \in 2^{\mathbb{N}}\right\} \subseteq G$ be a family such that for each $\tau \neq \sigma \in 2^{\mathbb{N}}$, there exists an integer $n_{\tau, \sigma} \geq 0$ such that

- $g_{\tau}(n)=g_{\sigma}(n)$ for all $n<n_{\tau, \sigma}$; and

- $g_{\tau}(n)^{-1} g_{\sigma}(n)$ is a noncentral element of $\mathrm{SL}\left(d, p_{n}\right)$ for all $n \geq n_{\tau, \sigma}$.

Since $[G: N] \leq \omega$, there exist $\tau \neq \sigma \in 2^{\mathbb{N}}$ such that $g_{\tau} N=g_{\sigma} N$ and hence $g=g_{\tau}^{-1} g_{\sigma} \in N$. Since $N$ is a normal subgroup, the conjugacy class $C=g^{G}$ is contained in $N$. Applying Proposition 4.3, it follows easily that $N$ contains the open subgroup $\prod_{n \geq n_{\tau, \sigma}} \mathrm{SL}\left(d, p_{n}\right)$ and hence $N$ is open.

Proof of Theorem 4.6. Let $G=\prod \operatorname{SL}\left(d, p_{n}\right)$ and let $\left\{P_{j} \mid j \in J\right\}$ be the set of open subgroups of $G$ such that $H \leq P_{j}$. Since $H \leq \bigcap_{j \in J} P_{j}$ and the intersection of infinitely many open subgroups of $G$ has index $2^{\omega}$, it follows that $J$ is finite. Let

$$
G^{\prime}=\prod_{n \geq n_{0}} \mathrm{SL}\left(d, p_{n}\right) \leq \bigcap_{j \in J} P_{j} .
$$

Then after replacing $G$ by $G^{\prime}$ and $H$ by its projection $H^{\prime}$ into $G^{\prime}$ if necessary, we can assume that $H$ is not contained in any proper open subgroups of $G$.

Let $G=\bigsqcup_{n \in \omega} g_{n} H$ be the coset decomposition of $H$ in $G$. Then we can construct a strictly increasing chain $H_{n}$ of proper subgroups of $G$ as follows.

- $H_{0}=H$.

- Suppose inductively that $H_{n}$ has been defined and that $H \leq H_{n}<G$. If $H_{n}$ is a maximal proper subgroup of $G$, then the construction terminates with $H_{n}$. Otherwise, let $k_{n}$ be the least integer $k$ such that $H_{n}<\left\langle H_{n}, g_{k}\right\rangle<G$ and let $H_{n+1}=\left\langle H_{n}, g_{k_{n}}\right\rangle$.

First suppose that there exists an integer $n$ such that $H_{n}$ is a maximal proper subgroup of $G$. Then we claim that $\left[G: H_{n}\right]=\omega$. Otherwise, $\left[G: H_{n}\right]<\omega$ and 
hence $N=\bigcap_{g \in G} g H_{n} g^{-1}$ is a normal subgroup of $G$ such that $N \leq H_{n}$ and $[G: N]<\omega$. Applying Lemma 4.7, it follows that $N$ is an open subgroup of $G$ and hence $H_{n}$ is also an open subgroup of $G$. But this contradicts the fact that $H$ is not contained in any proper open subgroups of $G$. Next assume that the construction does not terminate after finitely many steps and let $H_{\omega}=\bigcup_{n \in \omega} H_{n}$. Then either $H_{\omega}=G$ or else $H_{\omega}$ is a maximal proper subgroup of $G$. In the former case, $G$ has countable cofinality and hence, by Theorem 2.9, there exists a nonprincipal ultrafilter over $\omega$. Thus we can assume that $H_{\omega}$ is a maximal proper subgroup of $G$ and our earlier argument shows that $\left[G: H_{\omega}\right]=\omega$.

In order to simplify notation, we will assume that $H$ is a maximal subgroup of $G$. Hence, by considering the left translation action of $G$ on the set $\left\{g_{n} H \mid n \in \mathbb{N}\right\}$, we obtain a homomorphism

$$
\psi: G \rightarrow \operatorname{Sym}(\omega)
$$

such that $\psi(G)$ acts primitively on $\omega$. It follows that if $N \unlhd G$ is any normal subgroup, then either $\psi(N)=1$ or $\psi(N)$ acts transitively on $\mathbb{N}$. Let

$$
\mathcal{I}=\left\{A \subseteq \omega \mid \psi\left(\prod_{n \in A} \mathrm{SL}\left(d, p_{n}\right)\right)=1\right\}
$$

Then $\mathcal{I}$ is clearly an ideal on $\omega$. Furthermore, if $F \subseteq \omega$ is a finite subset, then $\psi\left(\prod_{n \in F} \mathrm{SL}\left(d, p_{n}\right)\right)$ cannot act transitively on $\mathbb{N}$ and so $F \in \mathcal{I}$. We will show that $\mathcal{I}$ is a prime ideal.

So assume that there exists a subset $A \subseteq \omega$ such that both $A \notin \mathcal{I}$ and $\omega \backslash A \notin \mathcal{I}$. Let $P=\prod_{n \in A} \mathrm{SL}\left(d, p_{n}\right)$ and let $Q=\prod_{n \in \omega \backslash A} \mathrm{SL}\left(d, p_{n}\right)$. Then both $\psi(P)$ and $\psi(Q)$ act transitively on $\mathbb{N}$. Suppose that $g \in P$ is such that $\psi(g)$ fixes some integer $n \in \mathbb{N}$. If $k \in \mathbb{N}$ is arbitrary, then there exists $h \in Q$ such that $\psi(h)(n)=k$; and since $g$ and $h$ commute, it follows that

$$
\psi(g)(k)=(\psi(g) \circ \psi(h))(n)=(\psi(h) \circ \psi(g))(n)=\psi(h)(n)=k .
$$

Thus $g \in \operatorname{ker} \psi$. It follows that $N=\operatorname{ker} \psi \cap P$ is a normal subgroup of $P$ such that $[P: N]=\omega$, which contradicts Lemma 4.7 .

Proof of Theorem 4.5. Let $G=\prod \mathrm{SL}\left(d, p_{n}\right)$ and assume that the symmetric countably syndetic subset $W \subseteq G$ witnesses the failure of the weak Steinhaus property. Let $H=\langle W\rangle$ be the subgroup generated by $W$. Then clearly $[G: H] \leq \omega$. If $[G: H]=\omega$, then the result follows from Theorem 4.6 and so we can assume that $[G: H]<\omega$. Applying Lemma 4.7, it follows easily that $H$ is an open subgroup of $G$. Let

$$
G^{\prime}=\prod_{n \geq n_{0}} \operatorname{SL}\left(d, p_{n}\right) \leq H
$$

and let $\pi: G \rightarrow G^{\prime}$ be the canonical projection. Consider the set $W^{\prime}=\pi(W)$ of generators of $G^{\prime}$. If $W^{\prime}$ witnesses that $G^{\prime}$ is not Cayley bounded, then the result 
follows from Theorem 2.9. Hence we can suppose that there exists an integer $k \geq 1$ such that $\left(W^{\prime}\right)^{k}=G^{\prime}$. Let $g=\left(g_{n}\right) \in G^{\prime}$ be such that $g_{n}$ is a noncentral element of $\mathrm{SL}\left(d, p_{n}\right)$ for all $n \geq n_{0}$. Then $g \in W^{\ell}$ for some $\ell \geq 1$; and Proposition 4.3 implies that

$$
G^{\prime} \subseteq \underbrace{W^{k} W^{\ell} W^{k} \cdots W^{k} W^{\ell} W^{k}}_{m \text { times }}=W^{2 k m+\ell m},
$$

where $m=8$ if $d=2$ and $m=2 d$ if $d>2$. But this contradicts the assumption that $W$ witnesses the failure of the weak Steinhaus property.

\section{The Bergman and Steinhaus Properties in $L(\mathbb{R})[\mathcal{U}]$}

In this section, we will present a partition property $P P$ for products of finite sets with measures; and we will show that $Z F+D C+P P$ implies that if $\left(H_{n} \mid n \in \omega\right)$ is a sequence of nontrivial finite groups such that $\left(d_{\mathbb{C}}\left(H_{n}\right) \mid n \in \omega\right)$ grows sufficiently fast, then $\prod H_{n}$ has both the Bergman property and the Steinhaus property.

The Partition Property. (PP) If $\left(\left\langle a_{n}, \mu_{n}\right\rangle \mid n \in \omega\right)$ is a sufficiently fast growing sequence of finite sets $a_{n}$ with measures $\mu_{n}$, then for every partition

$$
\prod a_{n}=\bigsqcup_{m \in \omega} X_{m}
$$

there exists an integer $m \in \omega$ such that $\prod b_{n} \subseteq X_{m}$ for some sequence of subsets $b_{n} \subseteq a_{n}$ such that $\lim _{n \rightarrow \infty} \mu_{n}\left(b_{n}\right)=\infty$.

Here the words "sufficiently fast growing" should be interpreted in the sense that there is a fixed function $f$ that assigns a natural number to every finite sequence of finite sets with measures $\left(\left\langle a_{m}, \mu_{m}\right\rangle \mid m<n\right)$ and that an infinite sequence $\left(\left\langle a_{n}, \mu_{n}\right\rangle \mid n \in \omega\right)$ is sufficiently fast growing if

$$
\mu_{n}\left(a_{n}\right)>f\left(\left(\left\langle a_{m}, \mu_{m}\right\rangle \mid m<n\right)\right)
$$

for all $n \in \omega$. The exact formula for the function $f$ is immaterial for the purposes of this paper. We will only mention that it is primitive recursive with a growth rate approximately that of a tower of exponentials of linear height. (The primitive recursive bound can be extracted from the argument in Shelah-Zapletal [30].)

The partition property $P P$ fails in $Z F C$, since the Axiom of Choice can be used to construct highly irregular partitions. However, it does hold in $Z F C$ if we restrict our attention to partitions into Borel sets; and it also holds for arbitrary partitions in many models of set theory in which the Axiom of Choice fails. In particular, in Sec. 7, we will prove the following result, which extends the work of Di Prisco-Todorcevic [8, Sec. 7].

Theorem 5.1. $(L C) L(\mathbb{R})[\mathcal{U}]$ satisfies $P P$.

We will also make use of the following recent result of Babai-Nikolov-Pyber [1] in the newly flourishing area of "arithmetic combinatorics". Recall that if $H$ is a 
nontrivial finite group and $K$ is a field, then $d_{K}(H)$ denotes the minimal dimension of a nontrivial $K$-representation of $H$; i.e. the least $d$ such that there exists a nontrivial homomorphism $\theta: H \rightarrow \mathrm{GL}(d, K)$.

Theorem 5.2. (Babai-Nikolov-Pyber [1]) Let $H$ be a nontrivial finite group and let $k$ be an integer such that $1 \leq k^{3} \leq d_{\mathbb{C}}(H)$. If $A \subseteq H$ is a subset such that $|A| \geq|H| / k$, then $A^{3}=H$.

Proof. By Babai-Nikolov-Pyber [1, Corollary 2.6], if $1 \leq k^{3} \leq d_{\mathbb{R}}(H)$ and $A \subseteq H$ with $|A| \geq|H| / k$, then $A^{3}=H$. Since $d_{\mathbb{C}}(H) \leq d_{\mathbb{R}}(H)$, the result follows.

Remark 5.3. If $H$ is a nontrivial finite group, then either $d_{\mathbb{R}}(H)=d_{\mathbb{C}}(H)$ or $d_{\mathbb{R}}(H)=2 d_{\mathbb{C}}(H)$. For the purposes of this paper, it does not matter whether we work with $d_{\mathbb{C}}(H)$ or $d_{\mathbb{R}}(H)$. Since most of the literature on the representation theory of finite groups deals with complex representations, we have chosen to state our results in terms of $d_{\mathbb{C}}(H)$.

Theorem 5.4. $(Z F+D C+P P)$ Let $\left(H_{n} \mid n \in \omega\right)$ be a sequence of nontrivial finite groups; and for each $n \in \omega$, let $k_{n}=\left\lfloor d_{\mathbb{C}}\left(H_{n}\right)^{1 / 3}\right\rfloor$ and let $\mu_{n}$ be the measure on $H_{n}$ defined by $\mu_{n}(A)=k_{n}\left(|A| /\left|H_{n}\right|\right)$. If $\left(\left\langle H_{n}, \mu_{n}\right\rangle \mid n \in \omega\right)$ grows sufficiently fast, then $\prod H_{n}$ has both the Bergman property and the Steinhaus property.

Proof. To see that $G=\prod H_{n}$ has the Steinhaus property, suppose that $W \subseteq G$ is a symmetric countably syndetic subset and let $G=\bigcup_{m \in \omega} g_{m} W$. Then $P P$ implies that there exists $m \in \omega$ such that $\prod A_{n} \subseteq g_{m} W$ for some sequence of subsets $A_{n} \subseteq H_{n}$ such that $\lim _{n \rightarrow \infty} \mu_{n}\left(A_{n}\right)=\infty$; and after replacing $\prod A_{n}$ by $g_{m}^{-1} \prod A_{n}$, we can suppose that $\prod A_{n} \subseteq W$. Let $n_{0} \in \omega$ be such that $\mu_{n}\left(A_{n}\right) \geq 1$ and hence $\left|A_{n}\right| \geq\left|H_{n}\right| / k_{n}$ for all $n \geq n_{0}$. Clearly we can suppose that $A_{n}=\left\{a_{n}\right\}$ is a singleton for each $n<n_{0}$. Applying Theorem 5.2, it follows that $W^{3} \supseteq g G^{\prime}$, where

- $g=\left(a_{0}^{3}, \ldots, a_{n_{0}-1}^{3}, 1,1, \ldots\right)$ and

- $G^{\prime}$ is the open subgroup $\prod_{n \geq n_{0}} H_{n}$.

Since $W$ is symmetric, it follows that $W^{6} \supseteq\left(g G^{\prime}\right)^{-1} g G^{\prime}=G^{\prime}$. This completes the proof that $\prod H_{n}$ has the Steinhaus property.

To see that $G=\prod H_{n}$ has the Bergman property, suppose that $G=\bigcup_{m \in \omega} U_{m}$ is the union of an increasing chain of symmetric subsets such that $U_{m} U_{m} \subseteq U_{m+1}$ for all $m \in \omega$. Arguing as above, it follows that there exists $m \in \omega$ such that $U_{m}^{6}$ contains an open subgroup $G^{\prime}$ and hence $G^{\prime} \subseteq U_{m+3}$. Since $\left[G: G^{\prime}\right]<\omega$, this implies that there exists $k \in \omega$ such that $G=U_{k}$, as required.

Unfortunately the statement of Theorem 5.4 is a little involved. However, we can obtain a much cleaner statement if we impose the following natural condition on the collection $\left\{G_{n} \mid n \in \omega\right\}$ of groups. 
Definition 5.5. A class $\mathcal{C}$ of nontrivial finite groups is said to be a Jordan class if for each integer $d \geq 1$, there exist only finitely many groups $H \in \mathcal{C}$ up to isomorphism such that $d_{\mathbb{C}}(H)=d$.

The following result is an immediate consequence of Theorem 5.4. Here the phrase "grows sufficiently fast" means that there is a function $f_{\mathcal{C}}: \mathbb{N} \rightarrow \mathbb{N}$ such that $\left(d_{\mathbb{C}}\left(H_{n}\right) \mid n \in \omega\right)$ grows sufficiently fast if $d_{\mathbb{C}}\left(H_{n+1}\right)>f_{\mathcal{C}}\left(d_{\mathbb{C}}\left(H_{n}\right)\right)$ for all $n \in \omega$. And the phrase has a similarly natural interpretation in the statements of Corollaries 5.7 and 5.8 .

Corollary 5.6. $(Z F+D C+P P)$ Let $\mathcal{C}$ be a Jordan class of nontrivial finite groups. If $H_{n} \in \mathcal{C}$ for all $n \in \omega$ and $\left(d_{\mathbb{C}}\left(H_{n}\right) \mid n \in \omega\right)$ grows sufficiently fast, then $\prod H_{n}$ has both the Bergman property and the Steinhaus property.

Corollary 5.7. (LC) If $d \geq 2$ and $\left(p_{n} \mid n \in \omega\right)$ is a sufficiently fast growing sequence of primes, then $\prod \mathrm{SL}\left(d, p_{n}\right)$ has both the Bergman property and the Steinhaus property in $L(\mathbb{R})[\mathcal{U}]$.

Proof. Recall that if $p \geq 5$ is a prime, then $d_{\mathbb{C}}(\mathrm{SL}(2, p))=(p-1) / 2$; and that if $d>2$, then $d_{\mathbb{C}}(\mathrm{SL}(d, p))=\left(p^{d}-p\right) /(p-1)$. Thus the result is an immediate consequence of Theorem 5.1 and Corollary 5.6.

It is perhaps worth pointing out that the proof of the following corollary does not make use of the classification of the finite simple groups.

Corollary 5.8. ( $L C)$ Suppose that $\left(H_{n} \mid n \in \omega\right)$ is a sequence of nonabelian finite simple groups. If $\left(\left|H_{n}\right| \mid n \in \omega\right)$ is sufficiently fast growing, then $\prod H_{n}$ has both the Bergman property and the Steinhaus property in $L(\mathbb{R})[\mathcal{U}]$.

Proof. By Jordan's theorem, there exists a function $\varphi: \mathbb{N} \rightarrow \mathbb{N}$ such that if $H$ is a finite subgroup of $\operatorname{GL}(n, \mathbb{C})$, then $H$ contains an abelian normal subgroup $N$ with $[H: N] \leq \varphi(n)$. (For example, see Curtis-Reiner [5, Theorem 36.13].) Thus the class $\mathcal{C}$ of nonabelian finite simple groups is a Jordan class; and if $\left|H_{n}\right|$ grows sufficiently fast, then $d_{\mathbb{C}}\left(H_{n}\right)$ also grows sufficiently fast.

We will conclude this section with a result which shows that it is necessary to impose some condition on the growth rate of the sequence $\left(d_{\mathbb{C}}\left(H_{n}\right) \mid n \in \omega\right)$ if we wish to obtain the conclusion of Theorem 5.4.

Theorem 5.9. $(Z F+D C)$ Suppose that there exists a nonprincipal ultrafilter over $\omega$. Then whenever $\left(H_{n} \mid n \in \omega\right)$ is a sequence of nontrivial finite groups such that $\lim \inf d_{\mathbb{C}}\left(H_{n}\right)<\infty$, then $\prod H_{n}$ does not have the automatic continuity property and hence does not have the Steinhaus property.

Proof. Recall that every complex representation of a finite group is similar to a unitary representation. (For example, see Curtis-Reiner [5, Exercise 10.6].) Hence 
there exist an infinite subset $I \subseteq \omega$ and a fixed integer $d \geq 1$ such that for each $n \in I$, there exists a nontrivial homomorphism $\varphi_{n}: H_{n} \rightarrow \mathrm{U}(d, \mathbb{C})$, where $\mathrm{U}(d, \mathbb{C})$ denotes the compact group of $d \times d$ unitary matrices. In order to simplify notation, we will assume that $I=\omega$.

For each $g_{n} \in H_{n}$ and $1 \leq i, j \leq d$, let $\varphi_{n}\left(g_{n}\right)_{i j}$ denote the $i j$ entry of the matrix $\varphi_{n}\left(g_{n}\right) \in \mathrm{U}(d, \mathbb{C})$. Then if $\mathcal{U}$ is a nonprincipal ultrafilter over $\omega$, we can define a homomorphism

$$
\begin{aligned}
\psi: \prod H_{n} & \rightarrow \mathrm{U}(d, \mathbb{C}) \\
\left(g_{n}\right) & \mapsto\left(z_{i j}\right),
\end{aligned}
$$

where $z_{i j}=\lim _{\mathcal{U}} \varphi_{n}\left(g_{n}\right)_{i j}$. We claim that $\psi$ is not continuous. To see this, suppose that $\psi$ is continuous and let $W \subseteq \mathrm{U}(d, \mathbb{C})$ be an open neighborhood of the identity element which contains no nontrivial subgroups. (For the existence of such a neighborhood, see Helgason [11, II.B.5].) Then there exists an open subgroup $H \subseteq \prod H_{n}$ such that $\psi(H) \subseteq W$ and hence $H \leq \operatorname{ker} \psi$. In particular, there exists a cofinite subset $A \subseteq \omega$ such that $\prod_{k \in A} H_{k} \leq \operatorname{ker} \psi$. For each $k \in A$, choose $g_{k} \in H_{k}$ such that $\varphi_{k}\left(g_{k}\right) \notin W$. Then, letting $g=\left(g_{k}\right) \in \prod_{k \in A} H_{k}$, we have that $\psi(g) \notin W$, which is a contradiction.

Remark 5.10. Recall that de Cornulier [6] has shown that if $G$ is a product of infinitely many copies of a fixed finite perfect group $H$, then $G$ has the Bergman property. Thus the analog of Theorem 5.9 is false for the Bergman property.

\section{The Bergman and Steinhaus Properties in $V$}

Suppose that $\left(H_{n} \mid n \in \omega\right)$ is a sequence of nonabelian finite simple groups. Then, in the previous section, assuming the existence of suitable large cardinals, we proved that if $\left(\left|H_{n}\right| \mid n \in \omega\right)$ is sufficiently fast growing, then $\prod H_{n}$ has both the Bergman property and the Steinhaus property in $L(\mathbb{R})[\mathcal{U}]$. In this section, we will briefly discuss the question of when $\prod H_{n}$ has either the Bergman property or the Steinhaus property in the actual set-theoretic universe $V$. In particular, throughout this section, we will work with the usual $Z F C$ axioms of set theory.

Recall that the classification of the finite simple groups says that if $S$ is a nonabelian finite simple group, then one of the following cases must hold.

(i) $S$ is one of the 26 sporadic finite simple groups.

(ii) $S$ is an alternating group $\operatorname{Alt}(n)$ for some $n \geq 5$.

(iii) $S$ is a group $L(q)$ of (possibly twisted) Lie type $L$ over a finite field $\mathbb{F}_{q}$ for some prime power $q$.

The following condition is the key to understanding when the product $\prod H_{n}$ has countable cofinality. 
Definition 6.1. A sequence $\left(H_{n} \mid n \in \omega\right)$ of nonabelian finite simple groups satisfies the Malcev condition if there exists an infinite subset $I$ of $\omega$ such that the following properties hold.

(a) There exists a fixed (possibly twisted) Lie type $L$ such that for all $n \in I$, $H_{n}=L\left(q_{n}\right)$ for some prime power $q_{n}$.

(b) If $n, m \in I$ and $n<m$, then $q_{n}<q_{m}$.

Arguing as in the proof of Theorem 2.7(a), it follows easily that if $\left(H_{n} \mid n \in \omega\right)$ satisfies the Malcev condition, then $\prod H_{n}$ has countable cofinality. Conversely, by Saxl-Shelah-Thomas [27, Theorem 1.9], if $\left(H_{n} \mid n \in \omega\right)$ does not satisfy the Malcev condition, then $\prod H_{n}$ has uncountable cofinality. Furthermore, as we mentioned earlier, Zalan Gyenis has recently checked that the arguments of Saxl-ShelahThomas [27] can be modified to prove that an infinite product $\prod H_{n}$ of finite simple groups has the Bergman property if and only if $\prod H_{n}$ has uncountable cofinality. Consequently, we have the following classification of the infinite products $\prod H_{n}$ of finite simple groups satisfying the Bergman property.

Theorem 6.2. If $\left(H_{n} \mid n \in \omega\right)$ is a sequence of nonabelian finite simple groups, then the following are equivalent:

(a) $\left(H_{n} \mid n \in \omega\right)$ does not satisfy the Malcev condition.

(b) $\prod H_{n}$ has the Bergman property.

The proof of Theorem 2.3 shows that if $\left(H_{n} \mid n \in \omega\right)$ satisfies the Malcev condition, then there exists a subgroup $K \leq \prod H_{n}$ with $\left[\prod H_{n}: K\right]=\omega$ and hence $\prod H_{n}$ does not have the Steinhaus property. Also, it is clear that if $\left(H_{n} \mid n \in \omega\right)$ satisfies the following condition, then $\prod H_{n}$ has a non-open subgroup of finite index and so once again the Steinhaus property fails. (See Example 1.1.)

Definition 6.3. A sequence $\left(H_{n} \mid n \in \omega\right)$ of nonabelian finite simple groups satisfies the Saxl-Wilson condition if there exists an infinite subset $I$ of $\omega$ and a fixed group $S$ such that $H_{n}=S$ for all $n \in I$.

In Thomas [31], it was shown that $\prod H_{n}$ has a non-open subgroup $K$ such that $\left[\prod H_{n}: K\right]<2^{\aleph_{0}}$ if and only if $\left(H_{n} \mid n \in \omega\right)$ satisfies either the Malcev condition or the Saxl-Wilson condition. Consequently, it seems natural to make the following conjecture.

Conjecture 6.4. If $\left(H_{n} \mid n \in \omega\right)$ is a sequence of nonabelian finite simple groups, then the following are equivalent:

(a) $\left(H_{n} \mid n \in \omega\right)$ satisfies neither the Malcev condition nor the Saxl-Wilson condition.

(b) $\prod H_{n}$ has the Steinhaus property. 
Remark 6.5. Using the classification of the finite simple groups, it is easily seen that condition (a) is equivalent to:

(a) $)^{\prime} \liminf d\left(H_{n}\right)=\infty$.

\section{The Partition Property (PP)}

Suppose that the Ramsey ultrafilter $\mathcal{U}$ is $L(\mathbb{R})$-generic for the notion of forcing $\mathcal{P}(\omega) /$ Fin. In this section, assuming the existence of suitable large cardinals, we will prove that the Partition Property $(P P)$ holds in $L(\mathbb{R})[\mathcal{U}]$. More specifically, we will make use of the following large cardinal assumption.

(LC) There exist infinitely many Woodin cardinals below a measurable cardinal.

Remark 7.1. It is perhaps worth pointing out that $L C$ is certainly not the minimal large cardinal assumption necessary to prove that $P P$ holds in $L(\mathbb{R})[\mathcal{U}]$. For example, it is enough to assume the statement $A_{\kappa}$ of Neeman-Zapletal [24] for a sufficiently large cardinal $\kappa$, and this is weaker in consistency strength than $L C$.

If we merely want to prove the consistency of $Z F+D C+P P$, then it is only necessary to assume the existence of an inaccessible cardinal. In more detail, suppose that $\kappa \in V$ is an inaccessible cardinal and that $\operatorname{Coll}(\omega,<\kappa)$ is the usual Lévy collapse. (For the basic properties of the Lévy collapse, see Jech [15, Chap. 26].) Let $G \subseteq \operatorname{Coll}(\omega,<\kappa)$ be a $V$-generic filter and let $\overline{\mathbb{R}}$ be the set of reals in the generic extension $V[G]$. Then the corresponding Solovay model $V(\overline{\mathbb{R}})$ consists of the sets $z \in V[G]$ which are hereditarily definable in $V[G]$ from parameters in $\overline{\mathbb{R}} \cup V$. Let the Ramsey ultrafilter $\overline{\mathcal{U}}$ be $V[G]$-generic for $\mathcal{P}(\omega) /$ Fin. Then clearly $\overline{\mathcal{U}}$ is also $V(\overline{\mathbb{R}})$-generic for $\mathcal{P}(\omega) /$ Fin. Most of our effort in this section will be devoted to proving the following result.

Theorem 7.2. With the above hypotheses, $V(\overline{\mathbb{R}})[\overline{\mathcal{U}}]$ satisfies PP.

To transfer this result from $V(\overline{\mathbb{R}})[\overline{\mathcal{U}}]$ to $L(\mathbb{R})[\mathcal{U}]$, we will make use of the fact that, assuming $L C$, if $\kappa \in V$ is the least inaccessible cardinal, then the theory of $L(\mathbb{R})[\mathcal{U}]$ is not altered by forcing with $\operatorname{Coll}(\omega,<\kappa)$. In more detail, assuming $L C$, the theory of $L(\mathbb{R})$ is not altered by forcing with $\operatorname{Coll}(\omega,<\kappa)$; i.e. $L(\overline{\mathbb{R}})$ is elementarily equivalent to $L(\mathbb{R})$. (For example, see Larson [20, Corollary 3.1.16].) Since $\mathcal{P}(\omega) /$ Fin is a homogeneous notion of forcing, it follows that if $\varphi$ is any sentence in the language of set theory, then

$$
\begin{aligned}
L(\mathbb{R})[\mathcal{U}] \vDash \varphi & \Longleftrightarrow L(\mathbb{R}) \vDash \mathcal{P}(\omega) / \text { Fin } \Vdash \varphi \\
& \Longleftrightarrow L(\overline{\mathbb{R}}) \vDash \mathcal{P}(\omega) / \text { Fin } \Vdash \varphi \\
& \Longleftrightarrow L(\overline{\mathbb{R}})[\overline{\mathcal{U}}] \vDash \varphi .
\end{aligned}
$$

Proof of Theorem 5.1. $(L C)$ Let $\kappa$ be the least inaccessible cardinal and let $V(\overline{\mathbb{R}})[\overline{\mathcal{U}}]$ be as in Theorem 7.2. Then the Ramsey ultrafilter $\overline{\mathcal{U}}$ is also $L(\overline{\mathbb{R}})$-generic 
for $\mathcal{P}(\omega) /$ Fin. Working inside $L(\overline{\mathbb{R}})[\overline{\mathcal{U}}]$, suppose that $\left(\left\langle a_{n}, \mu_{n}\right\rangle \mid n \in \omega\right)$ is a sufficiently fast growing sequence of finite sets $a_{n}$ with measures $\mu_{n}$ and that

$$
\prod a_{n}=\bigsqcup_{m \in \omega} X_{m}
$$

is any partition. Since $V(\overline{\mathbb{R}})[\overline{\mathcal{U}}]$ satisfies $P P$, there exists an integer $m \in \omega$ and a sequence of subsets $\left(b_{n} \subseteq a_{n} \mid n \in \omega\right) \in V(\overline{\mathbb{R}})[\overline{\mathcal{U}}]$ such that $\prod b_{n} \subseteq X_{m}$ and $\lim _{n \rightarrow \infty} \mu_{n}\left(b_{n}\right)=\infty$. Since $L(\overline{\mathbb{R}})[\overline{\mathcal{U}}]$ and $V(\overline{\mathbb{R}})[\overline{\mathcal{U}}]$ have the same reals, it follows that $\left(b_{n} \subseteq a_{n} \mid n \in \omega\right) \in L(\overline{\mathbb{R}})[\overline{\mathcal{U}}]$. Thus $L(\overline{\mathbb{R}})[\overline{\mathcal{U}}]$ satisfies $P P$. Finally since the theory of $L(\mathbb{R})[\mathcal{U}]$ is not altered by forcing with $\operatorname{Coll}(\omega,<\kappa)$, it follows that $L(\mathbb{R})[\mathcal{U}]$ also satisfies $P P$.

The remainder of this section will be devoted to the proof of Theorem 7.2. As usual, we will identify the notion of forcing $\mathcal{P}(\omega) /$ Fin with the quasi-order $\left([\omega]^{\omega}, \subseteq^{*}\right)$ of infinite subsets of $\omega$, quasi-ordered by $c \subseteq^{*} d$ if and only if $|c \backslash d|<\omega$. The key element of the proof is the work of Shelah-Zapletal [30] showing that for every sufficiently fast growing sequence $\left(\left\langle a_{n}, \mu_{n}\right\rangle \mid n \in \omega\right)$ of finite sets $a_{n}$ with measures $\mu_{n}$, there is a notion of forcing $\mathbb{P}$ with the following properties:

(1) $\mathbb{P}$ adds a new element $\dot{x} \in \prod a_{n}$.

(2) $\mathbb{P}$ is proper, ${ }^{\omega} \omega$-bounding and adds no independent reals.

(3) $\mathbb{P}$ is defined in a way which depends only on the reals; i.e. if $M \subseteq N$ are transitive models of set theory with the same reals, then $\mathbb{P}^{M}=\mathbb{P}^{N}$.

(4) Suppose that $M$ is a transitive model of set theory such that $\mathcal{P}(\mathcal{P}(\mathbb{R}))^{M}$ is countable. Then for every $p \in \mathbb{P}^{M}$, there exist a sequence of sets $\left(b_{n} \mid n \in \omega\right)$ with $b_{n} \subseteq a_{n}$ and $\mu_{n}\left(b_{n}\right) \rightarrow \infty$ such that the product $\prod b_{n}$ consists only of $M$-generic points for the poset $\mathbb{P}_{p}^{M}=\left\{q \in \mathbb{P}^{M} \mid q \leq p\right\}$.

Here an independent real is an infinite subset $a \subseteq \omega$ in the generic extension such that neither $a$ nor $\omega \backslash a$ contains an infinite ground model subset.

Let $\kappa \in V$ be an inaccessible cardinal and let $G \subseteq \operatorname{Coll}(\omega,<\kappa)$ be a $V$-generic filter. Suppose that $V(\overline{\mathbb{R}})$ is the corresponding Solovay model and that the Ramsey ultrafilter $\overline{\mathcal{U}}$ is $V[G]$-generic (and hence also $V(\overline{\mathbb{R}})$-generic) for $\mathcal{P}(\omega) /$ Fin. Let $\left(\left\langle a_{n}, \mu_{n}\right\rangle \mid n \in \omega\right) \in V(\overline{\mathbb{R}})[\overline{\mathcal{U}}]$ be a sufficiently fast growing sequence of finite sets $a_{n}$ with measures $\mu_{n}$ and let $\prod a_{n}=\bigsqcup_{m \in \omega} X_{m}$ be a partition of the product into countably many pieces within the model $V(\overline{\mathbb{R}})[\overline{\mathcal{U}}]$. Working inside $V[G]$, let $c_{0} \in[\omega] \omega$ be any infinite subset of $\omega$. Then it is enough to find a subset $c \in[\omega]^{\omega}$ with $c \subseteq^{*} c_{0}$ and a sequence of subsets $\left(b_{n} \subseteq a_{n} \mid n \in \omega\right) \in V[G]$ with $\lim _{n \rightarrow \infty} \mu_{n}\left(b_{n}\right)=\infty$ such that for some $m \in \omega$,

$$
c \Vdash \prod b_{n} \subseteq \dot{X}_{m} .
$$

Let $f: \prod a_{n} \rightarrow \omega$ be the function defined by

$$
f(x)=m \Longleftrightarrow x \in X_{m}
$$


and let $\dot{f} \in V(\overline{\mathbb{R}})$ be a $\mathcal{P}(\omega) /$ Fin-name for $f$. By the standard homogeneity arguments with respect to the Lévy collapse $\operatorname{Coll}(\omega,<\kappa)$, we can assume that $c_{0}$, $\left(a_{n} \mid n \in \omega\right) \in V$ and that the $\mathcal{P}(\omega) /$ Fin-name $\dot{f}$ is definable from the elements of the ground model $V$. In particular, it follows that there exists a formula $\varphi\left(v_{0}, v_{1}, v_{2}\right)$ with parameters in $V$ such that for every $V$-generic filter $H \subseteq \operatorname{Coll}(\omega,<\kappa)$ and every $c \in[\omega]^{\omega}, x \in \prod a_{n}$ and $m \in \omega$,

$$
V[H] \vDash c \Vdash_{\mathcal{P}(\omega) / \text { Fin }} \check{x} \in \dot{X}_{m} \Longleftrightarrow V[H] \vDash \varphi(c, x, m) .
$$

Working inside the ground model $V$, consider the product of the forcing $\mathbb{P}$ with $\mathbb{Q}=\mathcal{P}(\omega) /$ Fin. Then the poset $\mathbb{Q}$ adds a Ramsey ultrafilter $\mathfrak{u}$ and $\mathbb{P}$ adds a point $x \in \prod a_{n}$. Since the definition of the forcing $\mathbb{P}$ only depends on the real numbers, it follows that $\mathbb{P}^{V}=\mathbb{P}^{V[\mathfrak{u}]}$. Hence if $\mathfrak{u}, x$ are mutually generic, then $x$ will be $\mathbb{P}^{V[\mathfrak{u}]}$-generic over the model $V[\mathfrak{u}]$.

Lemma 7.4. In $V[\mathfrak{u}][x], \mathfrak{u}$ still generates a Ramsey ultrafilter.

Proof. By Shelah [29, VI.5.1], since $\mathbb{P}^{V}=\mathbb{P}^{V[\mathfrak{u}]}$ is proper and ${ }^{\omega} \omega$-bounding in $V[\mathfrak{u}]$, it is enough to show that $\mathfrak{u}$ still generates an ultrafilter in $V[\mathfrak{u}][x]$. First note that since $\mathbb{Q}$ is $\sigma$-closed and $\mathbb{P}$ is proper, it follows that $\mathcal{P}(\omega) \cap V[\mathfrak{u}][x]=\mathcal{P}(\omega) \cap V[x]$. (Since $\mathbb{P}$ is proper, each real $r \in V[\mathfrak{u}][x]$ is obtained from a countable collection $\mathcal{C}=\left\{C_{n} \mid n \in \omega\right\} \in V[\mathfrak{u}]$ of countable subsets $C_{n} \subseteq \mathbb{P}$ such that each $C_{n}$ is predense below some condition $p \in \mathbb{P}$; and since $\mathbb{Q}$ is $\sigma$-closed, it follows that $\mathcal{C} \in V$ and hence $r \in V[x]$.) Now suppose that $p \in \mathbb{P}, q \in \mathbb{Q}$ are conditions and that $p \Vdash \tau \subseteq \omega$. Since $\mathbb{P}$ does not add any independent reals, there exists a condition $p^{\prime} \leq p$ and an infinite subset $q^{\prime} \subseteq q$ such that either $p^{\prime} \Vdash q^{\prime} \subseteq \tau$ or $p^{\prime} \Vdash \tau \cap q^{\prime}=\emptyset$. Hence either $\left\langle q^{\prime}, p^{\prime}\right\rangle \Vdash \tau \in \dot{\mathfrak{u}}$ or $\left\langle q^{\prime}, p^{\prime}\right\rangle \Vdash \omega \backslash \tau \in \dot{\mathfrak{u}}$. It follows that $\mathfrak{u}$ still generates an ultrafilter in $V[\mathfrak{u}][x]$.

From now on, fix some $\mathfrak{u} \in V[G]$ such that $\mathfrak{u}$ is $V$-generic for $\mathbb{Q}$ and $c_{0} \in \mathfrak{u}$. Let $\mathbb{D} \in V[\mathfrak{u}]$ be the poset consisting of the conditions $(s, S)$, where $s \in[\omega]<\omega$ and $S \in \mathfrak{u}$, partially ordered by

$$
(s, S) \leq(t, T) \Longleftrightarrow s \supseteq t \text { and } s \backslash t \subseteq T .
$$

Then $\mathbb{D}$ adds an infinite subset $\dot{c} \subseteq \omega$ which diagonalizes the Ramsey ultrafilter $\mathfrak{u}$; i.e. a subset $\dot{c}$ such that $|\dot{c} \backslash S|<\omega$ for all $S \in \mathfrak{u}$. In fact, by Mathias [22], every set diagonalizing $\mathfrak{u}$ is $V[\mathfrak{u}]$-generic for the poset $\mathbb{D}$. By Lemma 7.4 , if $\overline{\mathfrak{u}}$ is the upwards closure of $\mathfrak{u}$ in the model $V[\mathfrak{u}][x]$, then $\overline{\mathfrak{u}}$ is a Ramsey ultrafilter in $V[\mathfrak{u}][x]$. Hence if $\overline{\mathbb{D}} \in V[x, \mathfrak{u}]$ is the corresponding poset diagonalizing $\overline{\mathfrak{u}}$, then $\mathbb{D}$ is dense in $\overline{\mathbb{D}}$ and every set diagonalizing $\mathfrak{u}$ is $V[\mathfrak{u}][x]$-generic for both $\overline{\mathbb{D}}$ and $\mathbb{D}$. Let $\varphi\left(v_{0}, v_{1}, v_{2}\right)$ be the formula with parameters in $V$ given by (7.3).

Lemma 7.5. In $V[\mathfrak{u}][x]$, there exists a natural number $m \in \omega$ such that

$$
\langle 1,1\rangle \Vdash_{\mathbb{D} \times \operatorname{Coll}(\omega,<\kappa)} \varphi(\dot{c}, \check{x}, \check{m}) ;
$$


in other words, in $V[\mathfrak{u}][x]$, we have that

$$
\langle 1,1\rangle \Vdash_{\mathbb{D} \times \operatorname{Coll}(\omega,<\kappa)} \quad \dot{c} \Vdash_{\mathcal{P}(\omega) / \text { Fin }} \check{x} \in \dot{X}_{m} .
$$

Proof. Suppose not. Then there exist distinct numbers $m_{0}, m_{1} \in \omega$ and conditions for the associated 3-step iteration

$$
\left\langle r_{0}, s_{0}, \dot{d}_{0}\right\rangle,\left\langle r_{1}, s_{1}, \dot{d}_{1}\right\rangle \leq\langle 1,1, \dot{c}\rangle
$$

such that $\left\langle r_{0}, s_{0}, \dot{d}_{0}\right\rangle \Vdash \check{x} \in \dot{X}_{m_{0}}$ and $\left\langle r_{1}, s_{1}, \dot{d}_{1}\right\rangle \Vdash \check{x} \in \dot{X}_{m_{1}}$. Choose mutually $V[\mathfrak{u}][x]$-generic filters $H_{0} \subseteq \mathbb{D}, K_{0} \subseteq \operatorname{Coll}(\omega,<\kappa)$ such that $\left\langle r_{0}, s_{0}\right\rangle \in H_{0} \times K_{0}$; and note that, since $d=\dot{d}_{0} /\left(H_{0} \times K_{0}\right) \subseteq{ }^{*} \dot{c} / H_{0}$, it follows that $d$ is $V[\mathfrak{u}][x]$-generic for the poset $\mathbb{D}$. Hence, after making a finite adjustment to the set $d$ if necessary, we can find a $V[\mathfrak{u}][x]$-generic filter $H_{1} \subseteq \mathbb{D}$ such that $r_{1} \in H_{1}$ and $d=\dot{c} / H_{1}$. A standard homogeneity argument with respect to the Lévy collapse $\operatorname{Coll}(\omega,<\kappa)$ now shows that there exists a $V[\mathfrak{u}][x]\left[H_{1}\right]$-generic filter $K_{1} \subseteq \operatorname{Coll}(\omega,<\kappa)$ such that $s_{1} \in K_{1}$ and $V[\mathfrak{u}][x]\left[H_{0} \times K_{0}\right]=V[\mathfrak{u}][x]\left[H_{1} \times K_{1}\right]$. Let $d^{\prime}=\dot{d}_{1} /\left(H_{1} \times K_{1}\right)$. Consider the notion of forcing $\mathcal{P}(\omega) /$ Fin inside the model $V[\mathfrak{u}][x]\left[H_{0} \times K_{0}\right]=V[\mathfrak{u}][x]\left[H_{1} \times K_{1}\right]$. Working with the $V[\mathfrak{u}][x]$-generic filter $H_{0} \times K_{0}$, it follows that $d \Vdash \check{x} \in \dot{X}_{m_{0}}$ and so $V[\mathfrak{u}][x]\left[H_{0} \times K_{0}\right] \vDash \varphi\left(d, x, m_{0}\right)$. Furthermore, since

$$
d^{\prime}=\dot{d}_{1} /\left(H_{1} \times K_{1}\right) \subseteq^{*} \dot{c} / H_{1}=d,
$$

it follows that $V[\mathfrak{u}][x]\left[H_{0} \times K_{0}\right] \vDash \varphi\left(d^{\prime}, x, m_{0}\right)$ and so $V[\mathfrak{u}][x]\left[H_{0} \times K_{0}\right] \not \models \varphi\left(d^{\prime}, x, m_{1}\right)$. On the other hand, working with the $V[\mathfrak{u}][x]$-generic filter $H_{1} \times K_{1}$, it follows that $d^{\prime} \Vdash \check{x} \in \dot{X}_{m_{1}}$ and so $V[\mathfrak{u}][x]\left[H_{1} \times K_{1}\right] \vDash \varphi\left(d^{\prime}, x, m_{1}\right)$, which is a contradiction.

Next, working in the model $M=V[\mathfrak{u}]$, let $p \in \mathbb{P}^{M}$ be a condition that identifies the natural number $m$ in the statement of Lemma 7.5. Then, since $\mathcal{P}(\mathcal{P}(\mathbb{R}))^{M}$ is countable in $V[G]$, there exists a sequence of sets $\left(b_{n} \mid n \in \omega\right) \in V[G]$ with $b_{n} \subseteq a_{n}$ and $\mu_{n}\left(b_{n}\right) \rightarrow \infty$ such that the product $\prod b_{n}$ consists only of $M$-generic points for the poset $\mathbb{P}_{p}^{M}=\left\{q \in \mathbb{P}^{M} \mid q \leq p\right\}$. Let $c \in V[G]$ be an infinite subset of $\omega$ which diagonalizes the ultrafilter $\mathfrak{u} \in M$. Then clearly $c \subseteq^{*} c_{0}$ and we claim that $c \Vdash \prod b_{n} \subseteq \dot{X}_{m}$. To see this, suppose that $x \in \prod b_{n}$. Then $\mathfrak{u}, x$ are mutually $V$-generic and $c$ is $V[\mathfrak{u}][x]$-generic for $\mathbb{D}$. Hence, working in $V[G]$, Lemma 7.5 implies that the condition $c \in \mathcal{P}(\omega) /$ Fin forces $\check{x} \in \dot{X}_{m}$. This completes the proof of Theorem 7.2.

\section{Acknowledgments}

Thanks are due to the referee for pointing out an inaccuracy in an earlier version of this paper and for making a number of suggestions that helped to improve the exposition. The research of the S. T. was partially supported by NSF Grants DMS 0600940 and DMS 1101597. The research of J. Z. was partially supported by NSF grant DMS 0801114 and Institutional Research Plan No. AV0Z10190503 and grant IAA100190902 of GA AV ČR. 


\section{References}

1. L. Babai, N. Nikolov and L. Pyber, Product growth and mixing in finite groups, in Proc. 19th Annual ACM-SIAM Symposium on Discrete Algorithms (ACM-SIAM, 2008), pp. 248-257.

2. G. M. Bergman, Generating infinite symmetric groups, Bull. London Math. Soc. 38 (2006) 429-440.

3. P. J. Cameron, Permutation Groups (Cambridge Univ. Press, 1999).

4. C. Chevalley, Introduction to the Theory of Algebraic Functions of One Variable (Amer. Math. Soc., 1951).

5. C. W. Curtis and I. Reiner, Representation Theory of Finite Groups and Associative Algebras (Wiley, 1962).

6. Y. de Cornulier, Strongly bounded groups and infinite powers of finite groups, Comm. Alg. 34 (2006) 2337-2345.

7. C. A. Di Prisco and S. Todorcevic, Perfect set properties in $L(\mathbb{R})[U]$, Adv. Math. 139 (1998) 240-259.

8. C. A. Di Prisco and S. Todorcevic, Souslin partitions of products of finite sets, Adv. Math. 176 (2003) 145-173.

9. M. Droste and W. C. Holland, Generating automorphism groups of chains, Forum Math. 17 (2005) 699-710.

10. E. W. Ellers, N. Gordeev and M. Herzog, Covering numbers for Chevalley groups, Israel J. Math. 111 (1999) 339-372.

11. S. Helgason, Differential Geometry, Lie Groups and Symmetric Spaces, Graduate Studies in Mathematics, Vol. 34 (Amer. Math. Soc., 2001).

12. J. E. Humphreys, Representations of SL(2,p), Amer. Math. Mon. 82 (1975) 21-39.

13. A. Ivanov, Strongly bounded automorphism groups, Colloq. Math 105 (2006) 57-67.

14. G. D. James, On the minimal dimensions of irreducible representations of symmetric groups, Math. Proc. Cambridge Philos. Soc. 94 (1983) 417-424.

15. T. Jech, Set Theory. The Third Millennium Edition, Revised and Expanded (SpringerVerlag, 2003).

16. A. S. Kechris and C. Rosendal, Turbulence, amalgamation, and generic automorphisms of homogeneous structures, Proc. Lond. Math. Soc. 94 (2007) 302-350.

17. J. Kittrell and T. Tsankov, Topological properties of full groups, Ergodic Theory Dynam. Systems 30 (2010) 525-545.

18. S. Koppelberg and J. Tits, Une propriété des produits directs infinis de groupes finis isomorphes, C. R. Math. Acad. Sci. Paris, Sér. A 279 (1974) 583-585.

19. S. Lang, Algebra, 2nd edn. (Addison-Wesley, 1984).

20. P. B. Larson, The Stationary Tower, Univ. Lecture Ser., Vol. 32 (Amer. Math. Soc., 2004).

21. H. D. Macpherson and P. M. Neumann, Subgroups of infinite symmetric groups, J. London Math. Soc. 42 (1990) 64-84.

22. A. R. D. Mathias, Happy families, Ann. Math. Logic 12 (1977) 59-111.

23. Y. N. Moschovakis, Descriptive Set Theory, 2nd edn., Mathematical Surveys and Monographs, Vol. 155 (Amer. Math. Soc., 2009).

24. I. Neeman and J. Zapletal, Proper forcing and L(R), J. Symbolic Logic 66 (2001) 801-810.

25. C. Rosendal and S. Solecki, Automatic continuity of homomorphisms and fixed points on metric compacta, Israel J. Math. 162 (2007) 349-371.

26. C. Rosendal, Automatic continuity of group homomorphisms, Bull. Symbolic Logic 15 (2009) 184-214. 
27. J. Saxl, S. Shelah and S. Thomas, Infinite products of finite simple groups, Trans. Amer. Math. Soc. 348 (1996) 4611-4641.

28. S. Shelah, On the cardinality of ultraproduct of finite sets, J. Symbolic Logic 35 (1970) 83-84.

29. S. Shelah, Proper and Improper Forcing, 2nd edn. (Springer-Verlag, 1998).

30. S. Shelah and J. Zapletal, Ramsey theorems for products of finite sets with submeasures, Combinatorica 31 (2011) 225-244.

31. S. Thomas, Infinite products of finite simple groups II, J. Group Theory 2 (1999) 401-434.

32. P. H. Tiep and A. E. Zalesskii, Minimal characters of the finite classical groups, Comm. Algebra 24 (1996) 2093-2167.

33. A. Wagner, The faithful linear representation of least degree of $S_{n}$ and $A_{n}$ over a field of characteristic 2, Math. Z. 151 (1976) 127-137.

34. A. Wagner, The faithful linear representation of least degree of $S_{n}$ and $A_{n}$ over a field of odd characteristic, Math. Z. 154 (1977) 103-114.

35. J. Zapletal, Forcing Idealized, Cambridge Tracts in Mathematics, Vol. 174 (Cambridge Univ. Press, 2008). 